\title{
Alianzas estratégicas y prácticas cooperativas que promueven la inclusión y el desarrollo territorial: Propuesta de un constructo de gobernanza cooperativa y territorial
}

\author{
Pedro Luís Büttenbender \\ Universidade Regional Noroeste do Estado do Rio Grande do Sul - ljuí - RS - \\ Brasil \\ ORCID: https://orcid.org/0000-0002-7011-8552 \\ Jorge Oneide Sausen \\ Universidade Regional Noroeste do Estado do Rio Grande do Sul - ljuí - RS - \\ Brasil \\ ORCID: https://orcid.org/0000-0003-3684-1410 \\ Bruno Nonnemacher Büttenbender \\ Universidade do Vale do Taquari - Lajeado - RS - Brasil \\ ORCID: https://orcid.org/0000-0001-9762-8977 \\ Ariosto Sparemberger \\ Universidade Regional Noroeste do Estado do Rio Grande do Sul - ljuí - RS - \\ Brasil \\ ORCID: https://orcid.org/0000-0001-5212-4695
}

\begin{abstract}
Resumen
El presente artículo tiene como objetivo estudiar prácticas cooperativas que puedan generar mayor inclusión social y económica en una región ubicada en la frontera internacional entre los países Argentina, Brasil y Paraguay, proponiendo un constructo de reflexión y análisis del proceso de gobernanza cooperativa y territorial. El estudio se caracteriza cuanto a los fines como un estudio multicaso, exploratorio y descriptivo, de naturaleza cualitativa y cuanto a los medios se caracteriza como una investigación de campo con uso de cuestionario semiestructurado aplicado a líderes y agentes sociales, agregado de una pesquisa bibliográfica, documental y de observación directa. La sistematización y análisis de los datos, relacionaron y contemplaron las evidencias empíricas identificadas, los fundamentos conceptuales, ponderaciones de distintos autores y interpretaciones conclusivas de los autores del estudio. Al final, el estudio ofrece una propuesta de constructo explicativo del proceso de gobernanza cooperativa y territorial.
\end{abstract}


Las cooperativas operan de acuerdo a las leyes de cada país y están influenciadas de varias otras características propias de las regiones de frontera internacional. Los avances del desarrollo territorial otorgan al cooperativismo una relevancia aún mayor para promover la creciente inclusión económica y social y la promoción de este. Las perspectivas del cooperativismo y el desarrollo indican priorizar el fortalecimiento del sistema cooperativo, calificación de la gestión y gobernanza, inversiones en la educación cooperativa, innovación productiva y tecnológica, y la conformación de nuevas alianzas estratégicas de intercooperación entre cooperativa y sociedad.

Palabras clave: Cooperativismo. Inclusión. Desarrollo. Territorios. Frontera.

\section{Strategic alliances and cooperative practices that promote inclusion and territorial development: Proposal for a cooperative and territorial governance construct}

\section{Abstract}

This article aims to study cooperative practices that can generate greater social and economical inclusion in a region located on the international border between Argentina, Brazil and Paraguay, proposing a construct for the reflection and analysis of the process of cooperative and territorial governance. The study is characterized according to its purposes, as a multicast, exploratory and descriptive study, of qualitative nature, and as for the means as field investigation using semi-structured questionnaire applied to leaders and social agents, aggregated from a bibliographic and documentary research and direct observation. The systematization and analysis of the data related and contemplated the empirical evidences identified, the conceptual foundations, considerations of different authors and conclusive interpretations of the study authors. In the end, the study offers the proposal of an explanatory construct for the process of cooperative and territorial governance. Cooperatives operate according to the laws of each country and are influenced by various other features of international border regions. Advances in territorial development give cooperativism even greater relevance for promoting the economic and social inclusion growing and its promotion. The perspectives of cooperativism and development indicate prioritizing the strengthening of the cooperative system, the qualification of management and governance, investments in cooperative education, productive and technological innovation and the formation of new strategic cooperative alliances between cooperatives and society.

Keywords: Cooperativism. Inclusion. Development. Territories. Border.

\section{Alianças estratégicas e práticas cooperativas que promovam a incluso e o desenvolvimento territorial: Proposta de um constructo de governança cooperativa e territorial}

\section{Resumo}

Este artigo tem como objetivo estudar práticas cooperativas que possam gerar maior inclusão social e econômica em uma região localizada na fronteira internacional entre os países Argentina, Brasil e Paraguai, propondo um construto para reflexão e análise do processo de governança cooperativa e territorial. O estudo caracteriza-se, quanto aos seus fins, como um estudo multicaso, exploratório e descritivo, de natureza qualitativa, e quanto aos meios como investigação de campo com uso de questionário semiestruturado aplicado a líderes e agentes sociais, agregado de uma pesquisa bibliográfica, documental e de observação direta. A sistematização e análise dos dados relacionaram e contemplaram as 
evidências empíricas identificadas, os fundamentos conceituais, ponderações de diferentes autores e interpretações conclusivas dos autores do estudo. No final, o estudo oferece a proposta de um construto explicativo para o processo de governança cooperativa e territorial. As cooperativas operam de acordo com as leis de cada país e são influenciadas por várias outras características das regiões fronteiriças internacionais. Os avanços no desenvolvimento territorial conferem ao cooperativismo uma relevância ainda maior para promover a crescente inclusão econômica e social e sua promoção. As perspectivas do cooperativismo e desenvolvimento indicam priorizar o fortalecimento do sistema cooperativo, a qualificação de gestão e governança, os investimentos em educação cooperativa, a inovação produtiva e tecnológica e a formação de novas alianças estratégicas de cooperação entre cooperativa e sociedade.

Palavras-chave: Cooperativismo. Inclusão. Desenvolvimento. Territórios. Fronteira.

\section{Introducción}

Las transformaciones en la sociedad, los nuevos y diferentes enfoques sobre las ciencias de la gestión del desarrollo, de la acumulación de competencias y de las competencias tecnológicas requieren nuevos aportes de conocimientos. Regiones que desean alcanzar niveles superiores de desarrollo, constituyéndose en territorios innovadores, requieren la acumulación de nuevas capacidades y competencias tecnológicas y sociales.

La presente tesis propone el estudio sobre los procesos de desarrollo de la región de la frontera, tomando como base la región Noroeste del estado de Rio Grande do Sul, proponiendo un modelo de gestión del desarrollo apuntando a la aceleración de estos procesos.

Los constantes cambios ocurridos en el proceso de desarrollo económico en las últimas décadas se caracterizan por la inestabilidad, el aumento de la competencia de los mercados y el crecimiento desigual entre las regiones. Para enfrentar estas adversidades y permitir el desarrollo económico, los diferentes agentes se han unido de diversas formas (cadenas productivas, acuerdos locales, alianzas estratégicas, redes, etc.). El objetivo de estas uniones, entendidas como acuerdos productivos, es alcanzar mejores niveles de competitividad que no tendrían condiciones de alcanzar de manera individual. Surgen de esta manera, nuevas formas de organización de la producción, las cuales pasan a ser percibidas como instrumentos de política industrial y regional.

Si por un lado, en la raíz del desarrollo regional, se encuentran diversas formas de organización de la producción; por el otro, existen diferentes teorías que buscan explicar científicamente la dinámica económica de una región. La teoría keynesiana, por ejemplo, describe los circuitos económicos en el ámbito nacional en un contexto de corto plazo. En cuanto al enfoque neoclásico, éste basa su análisis en mecanismos ajustados instantáneamente en función de las variaciones del mercado. El abordaje más reciente de los medios innovadores, tal vez sea el que más haya avanzado, ya que profundiza su estudio en cuestiones tales como capacidad de innovación y territorio.

El presente trabajo tiene como objetivo estudiar casos de prácticas cooperativas que generan mayor inclusión social y económica en una región de 
frontera internacional entre Argentina, Brasil y Paraguay, proponiendo un constructo que explique este proceso de gobernanza cooperativa y territorial. Y los objetivos específicos se estructuran como: a) Caracterizar la estructura económica y social de la Región Noroeste del Estado de Rio Grande do Sul, articuladas por los Consejos Regionales de Desarrollo - Coredes; b) Identificar las variables (instituciones, practicas innovadoras, tecnologías, visión emprendedora) determinantes en el proceso de desarrollo de la región; c) Identificar las acciones estratégicas que potencien los procesos de desarrollo de la región y los obstáculos que inhibieron los procesos de gestión del desarrollo de la región; d) Delimitar asimetrías y discrepancias que impactaron en la dinámica del desarrollo considerando las cuatro regiones coredianas del noroeste gaucho; e) A partir de un análisis, generar discusiones y contribuciones adicionales a los conceptos acerca de la gestión de los procesos de desarrollo y sus estructuras de gobierno; y f) Proponer un constructo de gobernanza cooperativa y desarrollo territorial sustentable y estrategias que puedan potenciar las alianzas estratégicas y la intercooperación en los procesos de gestión y de gobernanza del desarrollo y la inclusión económica y social.

\section{Referencial Conceptual}

El tema del desarrollo regional y de la gestión de los procesos de desarrollo es objeto de estudios, reflexiones y propuestas de diversas esferas de la sociedad. En la dimensión de los conceptos y paradigmas de desarrollo se constatan cambios y evolución en cuanto a su comprensión. Desde esta visión, el desarrollo, a partir del crecimiento económico, pasando por conceptos de desarrollo espacial y territorial hasta las comprensiones actuales, reconoce los aportes de diversas configuraciones políticas, sociales, económicas, tecnológicas, culturales y ambientales.

Las iniciativas de desarrollo en regiones o territorios protagonizadas por las demás estructuras e instituciones de la sociedad, tales como iglesias, cooperativas, organizaciones sociales y comunitarias, son evidenciadas como experiencias positivas y exitosas de desarrollo, basadas en estructuras de organización social y económica, referenciadas por el espíritu comunitario, cooperativo y emprendedor son conocidas como experiencias pioneras y positivas de desarrollo regional.

La verificación inicial del tema se fundamenta en la amplitud, complejidad y variedad de enfoques del desarrollo, como así también, el reconocimiento de los aportes y de múltiples influencias de impactos en el estudio y en la comprensión profunda del tema. El soporte de los procesos duraderos de desarrollo y que resulten en un desarrollo sustentable, requieren dinámicas de Gobernanza regional capaces de sustentar la participación y el compromiso de las estructuras del Estado, de agentes publico-gubernamentales, emprendedores, universidades y demás instituciones y organizaciones de la sociedad civil organizada (Veltz, 1995).

La conformación de una estructura de gobernanza regional de desarrollo se sustenta a partir de la verificación de una realidad empírica y de diferentes experiencias emprendedoras e innovadoras analizadas y estudiadas con base en fundamentos teórico-conceptuales y que resultan en la propuesta de un modelo de gestión del desarrollo de una región. 


\subsection{Gestion del desarrollo}

La temática del desarrollo regional y de la gestión del desarrollo ha sido objeto de reflexión constante entre los investigadores de diversas áreas, destacando las ciencias sociales y las aplicadas. Comprender la dinámica del desarrollo, los fenómenos ocurridos, el papel de los diferentes agentes implicados y los resultados prácticos de los procesos ha sido recurrente en la literatura de estas áreas.

La definición de una estrategia de desarrollo resulta del enfrentamiento de intereses diferenciados, lo que implica construir el desarrollo en múltiples escalas espaciales e instancias de poder (Brandão, 2007). Reforzando, Vainer (2002), afirma que cualquier proyecto o estrategia de transformación involucra, compromete y exige tácticas en cada una de las escalas en que actualmente se configuran los principales procesos sociales, económicos y políticos.

Una cuestión fundamental es la señalada por Fischer (2002): contemporáneamente, las relaciones entre los actores interorganizacionales e interinstitucionales, así como las estrategias de gestión, son transescalares. Además de otras implicaciones, de esto resulta que el abordaje y el enfrentamiento de los problemas deben ser, entonces, de naturaleza transescalar, o sea, implica actuar y trabajar en todas las escalas, no quedarse solamente en una escala localizada. Brandão (2007) complementa afirmando que, de esta manera, es imprescindible buscar construir estrategias multiescalares para el análisis de los fenómenos sobre los cuales se desea intervenir.

Tratar sobre desarrollo regional es, antes de cualquier cosa, pensar en la participación de la sociedad local, el planeamiento continuo de la ocupación del espacio y en la distribución de los frutos del proceso de crecimiento. La región es cada vez más uno de los puntos centrales de las estrategias que apuntan a la competitividad y el atractivo económico, y es en la región donde se refuerza la cohesión social. No obstante, se puede generar una mejor transversalidad en políticas públicas con la participación popular, y con esto, desarrollar una región, contando con la ayuda de instituciones y de liderazgos locales presentes en cada una de ellas (VEIGA, 2006). El desarrollo se caracteriza por el crecimiento (incrementos positivos en el producto y en la renta) representado por las transformaciones realizadas para satisfacer las más diversas necesidades del ser humano, tales como: salud, educación, habitación, transporte, alimentación, ocio, entre otras. Outos autores convergen con definiciones projimas, como Theis (2001), Sen (2000), Boisier (1996), Dallabrida (2011 y 2017), Veltz (1995) y Méndez (2006).

\subsection{Planeamiento Estratégico del desarrollo}

La gestión tiene la función de hacer que la organización alcance los objetivos a través de la utilización de todos los recursos, tanto humanos como materiales, a través del planeamiento, la organización, el liderazgo y el control. La gestión interpreta los objetivos propuestos por las organizaciones, así como de transformarlos en acción, o sea, concretarlos por medio del planeamiento, organización, dirección y control. 
La gestión comprende los procesos de planeamiento, liderazgo, organización y control de los esfuerzos realizados por los miembros de la organización y el uso de todos los otros recursos organizacionales para alcanzar los objetivos establecidos (DRUCKER, 1998). El define planeamiento como un proceso de establecimiento de objetivos. Siendo que los objetivos se caracterizan por los resultados específicos de las metas que se desean alcanzar. Define al planeamiento como una función administrativa que determina anticipadamente cuales son los objetivos que deben ser alcanzados y que se debe hacer para alcanzarlos. Complementa aun, que se trata de un modelo teórico para la acción futura.

El planeamiento estratégico hace referencia al relevamiento de la información fundamental para deliberar sobre las opciones consistentes con el fin de proponer acciones oportunas para el desarrollo de un determinado espacio (SIEDENBERG, BÜTTENBENDER Y ALLEBRANDT, 2010). Esto puede ser definido como la aplicación sistemática del conocimiento humano para prever y evaluar cursos de acción alternativos con vistas a la toma de decisiones adecuadas y racionales que sirvan de base para acciones futuras. No se trata solamente de un proceso técnico sino también político, siendo que abarca el encaminamiento de soluciones técnicas para el ámbito de la decisión política, así como la creación de actitudes de aceptación para las decisiones de planeamiento. La temática del desarrollo regional y de la gestión del desarrollo ha sido objeto.

\subsection{Gobernanza del Desarrollo Territorial}

Gobernanza es un término utilizado por diferentes áreas del conocimiento, principalmente, la sociología, la política, la administración, la economía y la geografía, ni siempre con el mismo sentido. La literatura reconoce que el concepto se origina en análisis sobre el mundo de las empresas. Se pasó a utilizar el término gobernanza para designar los dispositivos operacionalizados por la firma para acarrear coordinaciones eficaces, a los contratos y la aplicación de normas. Más tarde el término fue importado del campo empresarial para las discusiones sobre poder y organizaciones (MILANI y SOLINÍS, 2002, appud DALLABRIDA, 2011). Los autores Peters y Pierre (1998) destacan los principales elementos que se encuentran insertos en el debate conceptual sobre gobernanza, donde se priorizan tres: (1) el destaque de la importancia de las redes con el argumento de que las mismas pasaron a dominar la política pública; (2) relativización de la capacidad de control del Estado, argumentando que sustituye las formas de control directo por la capacidad de influencia en la negociación con los miembros de la red; (3) combinación de los recursos públicos y privados con la creación de sociedades más o menos formales entre los actores de gobierno y del sector privado en el proceso de gobernar.

De acuerdo con Fernandez et al (2008), en la temática del desarrollo territorial, el problema reside en un grupo de inconsistencias que ha dominado el cuerpo teórico del desarrollo regional, constituido en una "nueva ortodoxia" que orienta el contenido de las políticas de desarrollo territorial, tanto en los países centrales como periféricos. Para Dallabrida (2011) a gobernanza territorial acontece a través de la actuación de los diferentes actores, en las instituciones, incluyendo el Estado, y organizaciones de la sociedad civil, en redes de poder socioterritorial. 
Las relaciones de la gobernanza regional y territorial y las relaciones con el desarrollo y sus interfaces con el cooperativismo son profundizadas por Büttenbender (2014) y Nickel et al (2016).

\subsection{Gestión cooperativa y contribuciones al desarrollo}

En la Región Frontera Noroeste es significativa la presencia de cooperativas, en prácticamente todas las ramas del cooperativismo. En una nueva proposición de investigación, se efectuará un mapeamiento y diagnóstico general de las cooperativas presentes en la región Frontera Noroeste del RS. Büttenbender (2010 y 2011) aborda y describe varios estudios de caso sobre la actuación y la gestión de cooperativas en el Noroeste Gaúcho.

Las contribuciones de las cooperativas para el desarrollo de la región se exploran en diferentes estudios, destacando Nickel et al (2016) y Rotta et al (2016).

Estos estudios, protagonizados por Büttenbender (1995) y otros, orientados y inspirados en Benecke (1980), demuestran la importancia y las contribuciones de las cooperativas y de la organización asociativa para el desarrollo de la región. El estudio efectúa la inferencia directa de datos de 8 municipios, en el total de los 20 que componen la región Frontera Noroeste. El estudio detalla: la importancia y las contribuciones, además de la organización cooperativa, en el valor agregado generado por las cooperativas de estos municipios (contribuciones tributarias); como reguladores de mercado y de precios; como generadores de oportunidades de trabajo y renta; de fomento y extensión tecnológica a los productores; en el ofrecimiento de operatividad mercadológica, sea en la condición de oferta de bienes y servicios, sea en la demanda de bienes y servicios; ofrecimiento de servicios de crédito y operacionalidad bancaria; entre otros.

Estas constataciones corroboran con otras definiciones acerca de las contribuciones del cooperativismo al desarrollo regional, como destacadas por Büttenbender (2010 y 2011), cuando revela que las cooperativas generan aportes dinámicos la economía, el espíritu emprendedor y el desarrollo, como por ejemplo: las cooperativas son espacio de organización, participación, ejercicio de la democracia y de la ciudadanía entre sus miembros y la comunidad; las cooperativas son ambiente de inserción mercadológica, de operación y de agregación de valor a la cadena productiva y del trabajo, sea en la producción, la industrialización y el consumo; acceso a los mercados complejos, de grandes volúmenes y de negociación, ambientes que se consideran inaccesibles individualmente; las cooperativas, por la cooperación en el crédito, se fomentan el ahorro y el crédito, con la nueva inversión regional de las economías y recursos generados, inhibiendo transferencias de rentas y riquezas a otras regiones; las cooperativas operan mercados considerados menos dinámicos y geográficamente periféricos, y que son menos atractivos a los grandes conglomerados económicos; las cooperativas se constituyen en espacios de inclusión en el trabajo, con generación de oportunidades de trabajo, constituyéndose en escuelas de profesionalización para distintas actuaciones profesionales; y las cooperativas son espacios de inversión en la investigación, la ciencia y la tecnología, la innovación tecnológica, el fomento y la extensión tecnológica. 
Los niveles diferenciados de desarrollo para regiones con presencia de cooperativas, en detrimento de regiones con menor, o incluso sin, presencia de cooperativas es externado por Sescoop/RS (2018). Se demuestra el estudio que los municipios brasileños, en 2010, sin la presencia de cooperativas presentan un Índice de Desarrollo Humano - IDH de 0,706, mientras que los municipios que poseen la presencia de cooperativas, presentan un IDH de 0,731. Es decir, el estudio indica y sugiere que el desarrollo humano en los municipios y regiones con la presencia cualificada de cooperativas es un $3,5 \%$ superior a los municipios y regiones sin la presencia de cooperativas.

Otro fenómeno importante, involucrando el cooperativismo y el desarrollo, es su creciente presencia en estudios e investigaciones lideradas por las universidades y academias, con la realización de grandes eventos, destacándose los Encuentros Brasileños de Investigadores de Cooperativas, Encuentro Latinoamericano de Investigadores Cooperativos entre otros eventos nacionales e internacionales. Se destacan las elevadas inversiones en formación y educación cooperativa, ya sea a través de las acciones directas de las cooperativas, como los convenios y alianzas con universidades con la oferta de Cursos superiores y de postgrado dirigidos al área. Se destacan dos experiencias: La Escuela de Formación Profesional en Cooperativismo - ESCOOP, con sede en Porto Alegre / RS, con cursos de graduación y posgrado en el área; la experiencia de UNIJUI, Universidad ubicada en la región noroeste de Rio Grande do Sul, que ofrece un Curso Superior en Gestión de Cooperativas y un Curso de Postgrado - MBA en Gestión de Cooperativas. En la región no la educación cooperativa es accesible en todos los niveles de la educación, desde la creación hasta el postgrado. Se agregan a éstas, varias otras experiencias en otras regiones.

En el ámbito estadual, liderados por la Organización de las Cooperativas del Estado de RS y de Sescoop/RS (SESCOOP/RS, 2018), también se definieron siete grandes objetivos estratégicos: 1) enseñanza de formación profesional; 2) revitalización de los núcleos buscando la transparencia y el compromiso de los asociados con el emprendimiento cooperativo; 3) definir el foco de las cooperativas desvinculándolas de actividades ajenas a sus finalidades; 4) contractualización de las relaciones establecidas entre asociados y cooperativas; 5) asociaciones, creación o participación en centrales, incorporaciones y fusiones. 6) políticas públicas federales, estatales y municipales de apoyo al cooperativismo; y 7) mejora de los servicios del sistema Ocergs-Sescoop/RS.

El fortalecimiento de las cadenas productivas, generación de fortalecimiento de las oportunidades de trabajo y renta, por intermedio del cooperativismo, con mayor inclusión económica y social, son prioridades en el plan estratégico de desarrollo de la región Noroeste, como descrito por Büttenbender y Van Der Sand (2017): Inversiones en el fortalecimiento productivo, tecnológico y de agregación de valor a las cadenas productivas ; Inversiones en la infraestructura logística, energía e comunicación en la región; Inversiones en la cualificación tecnológica y de educación superior, visando la promoción de la investigación y desarrollo, integradas las cadenas productivas; y otros. Sin embargo, la región sólo puede aprovechar su proceso de desarrollo a partir del reconocimiento de su patrimonio histórico, social, político, económico, cultural y ambiental. En la medida en que la región se conoce a sí misma es capaz de recobrar las enseñanzas del pasado, 
descubrir sus potencialidades y proyectar colectivamente su futuro. El cooperativismo cumplió una misión importante en la historia de la región, sigue y seguirá representando esta misión en el desarrollo futuro, tanto de la región, del estado y de la nación.

\section{Metodología}

El estudio se caracteriza como un estudio de caso, exploratorio y descriptiva (Yin, 2001), de naturaleza cualitativa. Los autores Triviños (1987), Bryman (1988), Delgado y Gutiérrez (1995) y Taylor y Bogdan (1996) aclaran y sostienen que las investigaciones pueden ser al mismo tiempo cualitativas y cuantitativas.

El objetivo del estudio se basa en el análisis de la gestión de los procesos de desarrollo regional, con vistas a la propuesta de un modelo, tomando como base a la región Noroeste de Rio Grande do Sul. Estudios sobre desarrollo regional se sustentan en construcciones sociales, o sea, comportamientos, competencias, creencias, percepciones y valores. Por lo tanto, los métodos de investigación a ser empleados son originarios de esta perspectiva, considerando la observación directa, entrevistas cualitativas y la perspectiva participativa (EASTERBY-SMITH, 1991).

La presente investigación fue estructurada a partir de seis conjuntos de acciones, destacándose: 1) búsqueda de fundamentos y antecedentes en la literatura con interface en las experiencias, con el fin de delimitar el objetivo de la investigación y la problemática de la misma; 2) Elaboración y delimitación de los objetivos, la justificación y la temática y estructura central de la investigación; 3) La fundamentación teórico conceptual de los principales componentes y referencias necesarias para la realización del estudio; 4) Elaboración y definición de la tipología de investigación y sus procesos, con el fin de tener un sustento instrumental y metodológico de la investigación; 5) Delimitación y diagnostico de la información, datos e insumos necesarios para la descripción y análisis del objetivo del estudio; 6) La formulación de las propuestas prospectivas y estratégicas, en cuanto a los aportes de esta investigación, en la referencia, orientación y oferta de contribuciones al medio investigado y la propia sociedad, para atender la gestión y el gobernanza del proceso de desarrollo.

La sistematización y análisis de los datos, con la finalidad de atender a los objetivos del estudio, relacionaron y contemplaron las evidencias empíricas identificadas, los fundamentos conceptuáis, ponderaciones teóricas de distintos autores y interpretaciones conclusivas de los autores del estudio.

\section{Gobernanza cooperativa y territorial y la propuesta de un constructo}

La gestión del desarrollo regional parte del conocimiento de la amplitud, diversidad y complejidad de las relaciones e instituciones involucradas. La simplicidad sugiere una superficialidad en el soporte del proceso de desarrollo y de la sustentabilidad continua a lo largo del tiempo. En esta sección, luego de abordar la estructura y la dinámica de los consejos regionales de desarrollo, el perfil económico-social de la región noroeste del estado de Rio Grande do Sul, el capital institucional y el detalle de las principales cadenas productivas, se expone la 
perspectiva de presentar una propuesta de constructo para la gestión y el gobernanza territorial del desarrollo.

El enfoque y análisis de las asimetrías de las discrepancias que impactan en la dinámica del desarrollo, a partir de las capacidades de innovación, de fomento al emprendedorismo, el aumento de las competencias tecnológicas, económicas, sociales y ambientales y sus relaciones entre sí, constituyen las bases para la elaboración y propuesta de un constructo de gestión y de gobernanza innovador y territorial del desarrollo.

Diferentes autores han defendido recientemente que el desarrollo posee una fuerte relación con el estándar de organización socioeconómica territorial. Se destaca Velz (1995) cuando define que el desarrollo de los territorios, así como el desarrollo en general, pasa actualmente por la densidad y calidad de las relaciones entre los actores, dependiendo más de la pertinencia de los cuadros colectivos de acción, del vigor de los proyectos y de las anticipaciones del futuro que de la infraestructura y de los equipamientos.

Si es cierto que la innovación resulta de la lógica económica (modelo de organización territorial de la producción, configurado por la red empresarial local), la comprensión del proceso de innovación requiere, además de esta, la aprehensión de una lógica social (la dinámica institucional configurada por los actores que personifican las sociedades orientadas al desarrollo local), considerando que la innovación es esencialmente el resultado de un "proceso interactivo de aprendizaje colectivo" (LOPES, 2001).

Las sinergias de estos factores pueden generar innovaciones resultantes de la intensidad cualitativa de los factores locales de soporte a la competitividad (universidades, centros o institutos de investigación, escuelas técnicas, etc.), apoyando la constitución de territorios innovadores. La innovación como elemento esencial resultante de los procesos de aprendizaje individual y colectivo, deriva en la acumulación de nuevas competencias territoriales. El uso del término competencia territorial se justifica en el sentido etimológico que se expresa como la habilidad, la aptitud y la capacidad que posee un territorio para ofrecer mejores condiciones de vida a la población, conjugada con el enfoque geográfico.

Este proceso requiere también una elevada densidad institucional, capacidad de innovación colectiva con vistas al desarrollo de las cadenas de valor en el territorio. Como destaca Fernández (2003), un triangulo para habilitar nuevas estrategias de fomento al desarrollo y superar los limitados enfoques regionalistas del desarrollo.

En el estudio del entorno regional se constata la presencia del modelo del “Triangulo de Sábato", explorado y propuesto por Etzkowitz (2009), que presenta la cooperación entre el Estado (gobierno), la industria (empresarios) y la Universidad. La interface sistémica entre ambas resulta en el entorno de la "triple hélice", incluso en la Figura 1. El primer vértice, el Estado, comprende el conjunto de instituciones que tienen por finalidad formular e implementar políticas públicas y movilizar recursos hacia los otros dos vértices a través de procesos legislativos y administrativos. El segundo vértice, la industria (estructura productiva), abarca el conjunto de sectores productivos que proveen los bienes y servicios demandados por la sociedad. El tercer vértice, la Universidad (infraestructura científicotecnológica), comprende las siguientes dimensiones: a) el sistema educativo que 
forma a los individuos que protagonizan las actividades de investigación (científicos, tecnólogos, administradores); b) los laboratorios, institutos y centros de P\&D; c) el sistema de planeamiento, promoción, coordinación y estimulo a la investigación (consejos de investigación, academias de ciencias); y d) los mecanismos jurídicoadministrativos que regulan las instituciones de investigación y los recursos financieros aplicados a su funcionamiento.

Inicialmente indicado para países desarrollados, en el enfoque de los temas de la gestión del conocimiento, del desarrollo y de la transferencia de tecnología, pasó a ser aplicable a la realidad y entorno de los países y regiones en desarrollo. Este modelo indica que la Universidad (academia) asume un posicionamiento dinámico, capaz de promover la cooperación y la competitividad de las organizaciones (empresas) en entorno en la región. El Estado que se ubica en el vértice superior del "Triangulo de Sábato" establece una relación mutua con la Academia, ya que algunas actividades u objetivos se superponen. Este vinculo es complementario a la relación Universidad-Empresa, constituyendo, de esta manera, una relación triple: universidad, empresas y gobierno (DAGNINO,2003).

La gestión del desarrollo de la región Noroeste de RS, con base en el sistema de cooperación de la triple hélice, sugiere la necesidad de un enfoque más amplio, que incorpore la trayectoria de las interfaces entre las políticas y programas de innovación y de transferencia tecnológica. Se constata una frágil estructura de inversión articulada con las estrategias consolidadas. La existencia de una red institucional relevante basada en organismos gubernamentales, consejos, universidades, polos de innovación tecnológica, agencias de desarrollo, sindicatos, etc., con reconocida cantidad de programas y proyectos, no se presentan con un enfoque e intensidad suficientes para apalancar una trayectoria sustentable de desarrollo.

Las iniciativas existentes, a pesar de relevantes y altamente oportunas, son aisladas y reflejan de forma limitada la interface de inversión de recursos publicoestatales, el enfoque y el énfasis de los proyectos en el ámbito de las universidades y de las demandas de las empresas y de los procesos productivos. La ausencia de un plan estratégico de desarrollo científico y tecnológico para la región Noroeste sostiene la propuesta y mantenimiento de iniciativas importantes, pero aisladamente insuficientes. Combinados, las tímidas inversiones en investigación y desarrollo por parte de las empresas, revelan la comprensión limitada de la importancia de la innovación. Aun cuando se instalan procesos de aprendizaje más intensos y ocurre la innovación y la acumulación de nuevas competencias tecnológicas, estas se encuentran distantes de la tecnología de frontera de la respectiva industria y de agregados significativos en la performance técnica y económica de las firmas. Estas definiciones corroboran también estudios anteriores volcados hacia la industria metalmecánica, fabricante de maquinas agrícolas, como publicado por Büttenbender (2008 y 2010).

Etzkowitz (2009) lanza una advertencia que converge con el entorno regional diagnosticado, donde la innovación debe considerar el desarrollo de nuevos productos en las empresas, pero también la creación de nuevos acuerdos entre las esferas institucionales que propicien las condiciones para la innovación. El diagnostico regional, evidenciado durante la recolección de los datos, revela que los vértices de este triangulo se encuentran desconectados. La aproximación, 
alineación, integración y cooperación de estas instituciones es señalada como una posible solución para la cuestión de la innovación tecnológica, pues presupone una integración directa entre el Estado, la ciencia realizada en la Universidad y la tecnología desarrollada en la Industria (empresas).

La existencia de aglomerados productivos aun incipientes, como por ejemplo los acuerdos productivos locales - APLs, demuestra una fragilidad en la promoción del aprendizaje colectivo e interorganizacional. A partir de la delimitación de las asimetrías y discrepancias que impactan en el proceso de desarrollo de la región, se constituyen las bases para la formación de territorios innovadores, con base en el emprendedorismo y en la economía del conocimiento, que sugieren la combinación de inversiones en tecnología, innovación y emprendedorismo. La combinación triangular, bajo el punto de vista de Julien (2010), reconoce las relaciones intra (dentro de cada vértice), inter (entre los tres vértices) y extra (entre cada uno de los vértices con el contexto externo del espacio en el cual se encuentran).

La región revela las inversiones necesarias en estructuras calificadas de investigación y desarrollo que prioricen la acumulación de competencias tecnológicas volcadas hacia las cadenas productivas. La elevación de los niveles de las funciones tecnológicas con la capacidad de realizar funciones tecnológicas más avanzadas y compelas se incorpora como una prioridad. Aportando nuevos procesos de aprendizaje con una mayor intensidad e interacción incorporaran nuevas competencias y capacidades de innovación como define Figueiredo (2009). A partir de los procesos de aprendizaje, con el aumento de las capacidades para generar soluciones tecnológicas (funciones tecnológicas) y la optimización de las competencias para concebir innovaciones sugieren inversiones en mejora del entorno emprendedor. Como señala Julien (2010), la constitución de oportunidades y niveles diferenciados de creación de valor dependerá de la capacidad de generar innovación y rupturas en los mercados.

Desarrollar entornos favorables y con competencias para transformar las capacidades tecnológicas, productivas y de innovación en oportunidades para agregar trabajo e ingresos. Emprender nuevos negocios en cualquiera de los dos eslabones de las cadenas productivas, ya sea de manera autónoma o insertos en otros sistemas de producción. La red económica y productiva requiere el estimulo a las capacidades emprendedoras, aumentando y mejorando el agregado de valor y la generación de nuevas oportunidades de trabajo e ingreso. Con este fortalecimiento económico, con equilibrio ambiental y equidad social, se realimentarán los procesos de aprendizaje con vistas a la acumulación de nuevas competencias tecnológicas y las capacidades de innovación.

En el enfoque del constructo de la matriz de desarrollo, del progreso del territorio, se incorpora el tercer conjunto de variables que son los pilares del desarrollo sustentable, de acuerdo con lo propuesto por Elkington (1998) y Días (2009): económico, social y ambiental. Los pilares son también explorados por Sachs (2004), como cinco elementos, agregando las variables políticas y territoriales. Sin ser tautológico, en cuanto los enfoques anteriores, esta matriz aplicada a la realidad regional estudiada, analizan los tres pilares sugiriendo el desarrollo económico eficiente, equidad social y equilibrio ambiental, en conformidad con la combinación que se ilustra en la Figura 1. 
Se refuerzan en este enfoque los aportes realizados por Veiga (2006), en cuanto sintetiza que el desarrollo sustentable combina cuatro indicadores: a) llevar una vida larga y saludable; b) ser instruido; c) tener acceso a los recursos necesarios para un nivel de vida digno; y d) ser capaz de participar de la vida en comunidad. Definiciones que se corroboran con las contribuciones de Almeida (2007), cuando argumenta que el desarrollo sustentable se relaciona con las estructuras de poder y sus interfaces entre gobiernos, empresas y organizaciones de sociedad civil.

El desarrollo sustentable de la región Noroeste de RS apunta hacia las estructuras de gobernanza del desarrollo regional y de forma más específica, como define Dallabrida (2011) a la estructura de gobernanza socio territorial. La combinación de estrategias, basadas en un plan estratégico de desarrollo, que contemplan las dinámicas del gobernanza regional, son capaces de sostener la participación y el compromiso de las estructuras del Estado, agentes publicogubernamentales, emprendedores, universidades y demás instituciones y organizaciones de la sociedad civil organizada. La definición coincide con Veltz (1995) cuando señala que el proceso de desarrollo sustentable del territorio requiere: a) de la competencia de sus cuadros colectivos de acción y liderazgo; b) de la competencia local en la constitución de redes internas y externas, de diferentes formas de organización social local; c) de la capacidad de construir en consenso una visión de futuro; y d) de la densidad y calidad de la interacción entre los liderazgos con la presencia de instituciones solidas y activas.

El análisis de contexto regional bajo estudio sugiere un enfoque integrado y sistémico de los tres modelos abordados anteriormente: a) la Gestión del Desarrollo con base en el "Triangulo de Sábato" (Estado, Industria y Universidad); b) la Gestión del Desarrollo con base en los territorios innovadores (Tecnología, Innovación y Emprendedorismo); y c) los Pilares del Desarrollo Sustentable (económico, social y ambiental). La formulación de un nuevo constructo que relaciona sistemáticamente los tres grupos de modelos, donde cada conjunto en particular anteriormente detallado revela la combinación triangular que reconoce las relaciones intra (dentro de cada vértice), inter (entre los tres vértices) y extra (entre cada uno de los vértices con el contexto externo del espacio en el cual se encuentran).

La exploración justificada del tema del desarrollo de la región, del territorio del noroeste gaucho sugiere la necesidad de reconocer el conjunto de antecedentes, de las referencias conceptuales, de la complejidad de los temas, la amplitud de las relaciones involucradas y la acumulación de competencias estratégicas y tecnológicas superiores. El abordaje individual de cada modelo tendera a un enfoque lineal y simplificado frente a la amplitud del tema. Por lo tanto, asociados al contexto de su trayectoria histórico-evolutiva, la amplitud de las relaciones y la variedad de factores involucrados para el desarrollo sustentable de una región mediterránea, presenta desafíos adicionales, lo que sugiere un abordaje combinado, sistémico e interdependiente, proponiendo la constitución de un nuevo constructo de gobernanza innovador y territorial del desarrollo, como lo muestra la Figura 1.

En el análisis del constructo, además de identificar de manera objetiva la combinación de los tres conjuntos, en el cual cada uno se fundamenta en una conceptualización propia, es posible verificar e identificar la combinación triangular, sinérgica e integrada, formando una propuesta innovadora y diferenciada de 
constructo de análisis y de gestión de los procesos de desarrollo regional y territorial.

El constructo propuesto se reporta al papel y a la importancia de los líderes gestores de los procesos de desarrollo, atribuyendo asimetrías entre la importancia y las relaciones de causa y efecto. Coincide con el modelo del triangulo de la sustentabilidad (modelo 3 D's) propuesto por Mauerhofer (2008), sin, no obstante, debilitar con la atribución de los grados de importancia y de los paradigmas disciplinares. Los procesos, etapas y competencias de la gestión, inherentes a la trayectoria de desarrollo de regiones y territorios, podrá agregar importantes aportes de otros autores.

En el ámbito de las ciencias sociales aplicadas, incorporar la responsabilidad de proponer un constructo de gobernanza cooperativa para el desarrollo territorial sustentable y reconocer la posibilidad de su refutabilidad, bajo el punto de vista del filósofo Karl Popper (1982), y no podrá ser considerada una verdad absoluta o definitiva.

Figura 1 - Propuesta de constructo de Gobernanza Cooperativa para el Desarrollo

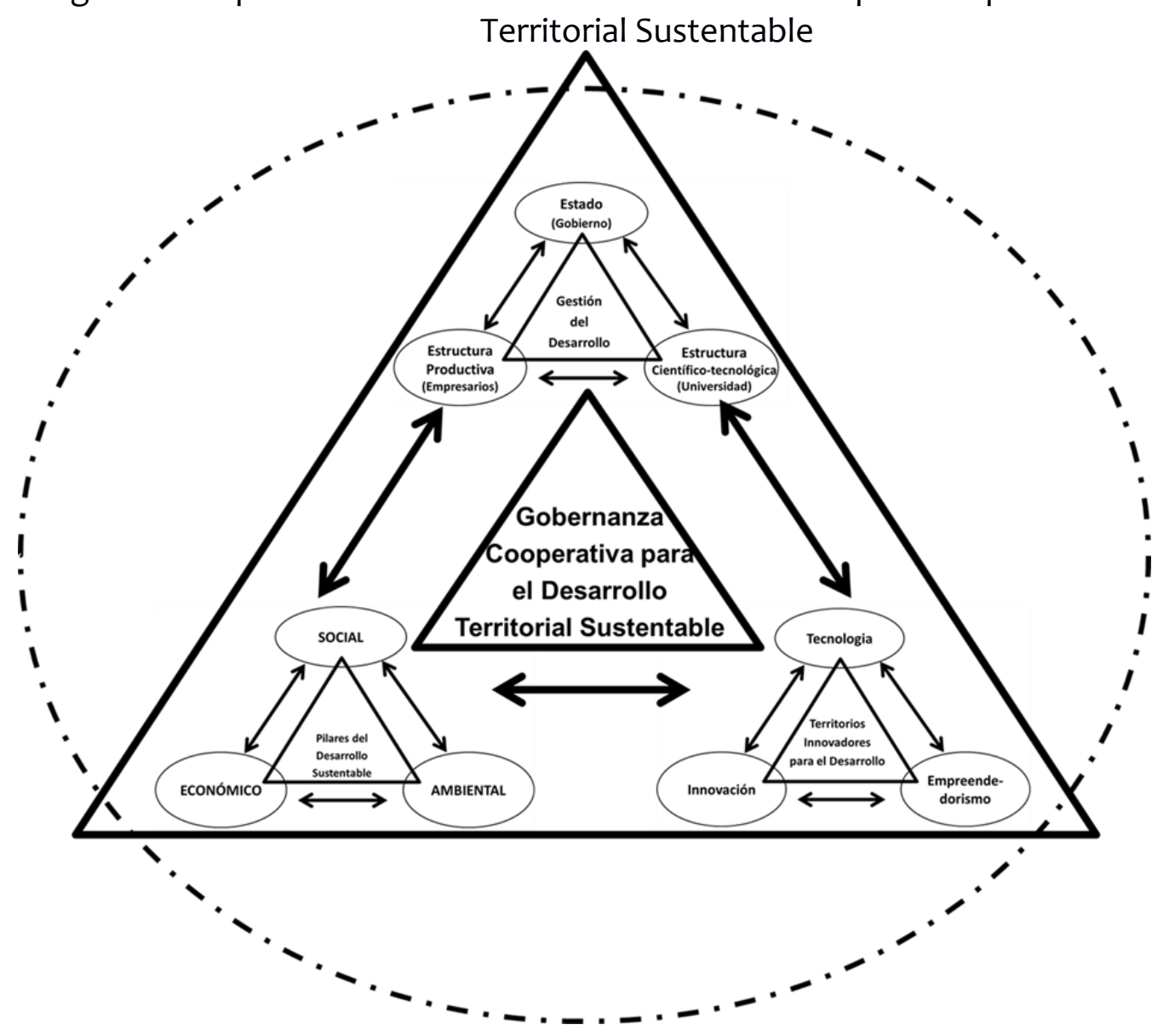

Fuente: Elaboración de los autores, adaptado de Büttenbender (2014)

El proceso de desarrollo a partir de la propuesta de un constructo de gobernanza cooperativa para el desarrollo territorial sustentable concierne a un conjunto de estrategias que puedan potenciar las iniciativas prometedoras en los procesos de gestión y de gobernanza del desarrollo de la región, del territorio. 
Como se ha detallado en el estudio, los conceptos sobre el desarrollo varían a lo largo del tiempo y se sustentan en diferentes escuelas y que se reflejan en el ámbito del territorio bajo estudio: estrategia de modernización en la década de 1950; estrategia de la disociación en la década de 1960; estrategia de evaluación de necesidades básicas en la década de 1970; estrategia del ajuste natural en la década de 1980; estrategia del desarrollo sustentable en la década de 1990; estrategia de gobernanza global a finales de los años 1990; y las estrategias de mediano alcance, de los pactos socio-territoriales y constitución de regiones innovadoras, a partir del 2000. En este contexto se establecieron elementos constitutivos del desarrollo y que destacan a las regiones que sean capaces de invertir e integrar a la investigación, la ciencia y la tecnología con los sistemas productivos del territorio.

Las bases del desarrollo sustentable en la sociedad del conocimiento se identifican en las estrategias enfocadas en las redes de cooperación que involucran al poder público (estructura del Estado en todos sus niveles), empresas (emprendedores), las universidades (centros de investigación y desarrollo, escuelas en todos sus niveles) y las organizaciones comunitarias (iglesias, ONG's, asociaciones, cooperativas, sindicatos, etc.).

La dinámica de la globalización o mundialización de las competencias estratégicas y tecnológicas apunta hacia el territorio en la medida en que el proceso de globalización condiciona la dinámica económica de territorios/regiones/ciudades/lugares. Como los territorios son impactados por los actores locales estableciendo una dinámica territorial de desarrollo, constituyendo territorios innovadores y que potencian sus competencias de agregado de valor, generar oportunidades de trabajo e ingreso, incorporando nuevas tecnologías a los sistemas productivos.

Frente a las asimetrías y discrepancias que impactan en la dinámica del desarrollo del territorio bajo estudio y los componentes del constructo propuesto, se proponen estrategias que puedan potenciar las iniciativas prometedoras del desarrollo:

1. Composición de un pacto socio-territorial regional cooperativo, abarcando liderazgos del poder público (estructura del Estado en todos sus niveles), empresas (emprendedores), las universidades (centros de investigación y desarrollo, escuelas en todos los niveles), y las organizaciones comunitarias (iglesias, ONG's, asociaciones, cooperativas, sindicatos, etc.), articuladas por los Consejos Regionales de Desarrollo - Coredes.

2. Estrategia de aceleración del desarrollo a través de la calificación, modernización y fortalecimiento de la gestión pública, con una mayor integración entre las políticas públicas, programas y proyectos de los diversos entes federales (federal, estatal y municipal) en la región.

3. Estrategias de fortalecimiento cooperativo, extensión tecnológica y productiva, innovación, cooperación entre las cadenas productivas presentes en la región con las estructuras de ciencia y tecnología, universidades, polos de innovación tecnológica y centros de generación, difusión y extensión tecnológica.

4. Proyecto de inversión en expansión y modernización de infraestructura logística, de comunicación y tecnológica.

5. Promover el emprendedorismo cooperativo tecnológico-territorial, priorizando iniciativas productivas de elevada performance tecnológica y de valor, 
estimulando la formación de alianzas y redes locales de cooperación, intercooperación y redes internacionales de cooperación en función del desarrollo y la acumulación de competencias productivas y la inclusión económica y social.

6. Ampliar inversiones en los procesos de educación y formación técnicoprofesional e en cooperativismo, en todos los niveles de la sociedad, combinando y potenciando iniciativas que vienen siendo emprendidas en la región.

7. Ofrecer propuestas de desarrollo de regiones mediterráneas, orientados por los mecanismos de participación y concentración regional, políticas públicas, programas, proyectos y recursos de inducción y de discriminación positiva de procesos de desarrollo.

8. Elevar los niveles de atractivo de emprendimientos e inversiones, de formación y retención de profesionales con competencias tecnológicas e innovadoras, constituyendo un ambiente emprendedor y un entorno territorial innovador.

Las estrategias propuestas requieren el complemento de programas, proyectos y acciones de la región basadas en el planeamiento y en la gestión estratégica del desarrollo regional con la activa participación de las cooperativas. La concreción de la dinámica que permite la aceleración de los procesos de desarrollo sugiere la elaboración de planes estratégicos de desarrollo. Estos planes se encuentran articulados y alineados en distintas combinaciones. En las relaciones horizontales, integrando regiones y territorios. En la dinámica piramidal, vertical, integrando y alineando planes estratégicos municipales y territorios y estos con las esferas estatal, federal e internacional. En las relaciones transversales, los planes estratégicos deben producir integración y alineación entre las prioridades y demandas del poder público; de las iniciativas productivo-empresariales; de las universidades y estructuras de ciencia y tecnología; y de la sociedad organizada. De igual manera requieren nuevas alianzas estratégicas entre las cooperativas, promoviendo la intercooperación.

Las estrategias a lo largo del periodo incorporarán nuevos desafíos y cambios y reconocerán nuevas variables que están más allá de los objetivos del presente estudio, lo que deberá motivar la mejora continua, la revisión periódica, manteniendo la actualización y pertinencia de las estrategias y prioridades. Este reconocimiento sugiere la promoción de un sistema de gestión y de gobernanza innovador y territorial del desarrollo.

\section{Consideraciones finales}

Los avances, innovaciones y cambios en la sociedad y en las organizaciones impactan en las diversas concepciones y conceptos de la gestión. Los procesos de desarrollo generan prácticas cooperativas de promoción, gestión e de gobernanza cooperativa y intercooperativa. Representar e identificar las variables y sus impactos, combinados con la competencia estratégica y tecnológica de liderar e implementar iniciativas que promuevan el desarrollo sustentable de una región o territorio constituye uno de los pilares diferenciadores. 
El problema del estudio de esta investigación se fundamentó en el desafío de relacionar la evolución de las trayectorias de acumulación de competencias tecnológicas, la innovación, el emprendedorismo como proceso de gobernanza del desarrollo territorial. La reflexión propositiva a partir de los fundamentos teóricos, la experiencia empírica de los temas junto a las organizaciones, la universidad y la región generaron la posibilidad de aportar percepciones originales, diferenciadas y propias y proponer un constructo de gobernanza cooperativa para el desarrollo territorial sustentable.

A partir de los aportes generados, ofrece la propuesta de un constructo de gobernanza innovador y territorial del desarrollo. No obstante, los fundamentos y las bases del constructo permiten su réplica en los estudios de otras regiones y territorios. Nuevas motivaciones de investigación se generan a partir del presente estudio, lo que reconoce la amplitud y la naturaleza del mismo.

O ámbito de las cooperativas, las as relaciones sociales, económicas, culturales y ambientales están fuertemente influenciadas por la dinámica de territorios de frontera internacional, asumiendo impactos en la inclusión social y económica de su gente. El desarrollo territorial de esta región frontera solamente puede ser entendido y explicado, conociendo y reconociendo la importancia, en la trayectoria histórica y evolutiva, de los aportes del cooperativismo. Considerando los avances y transformaciones económicas, sociales, tecnológicas y ambientales de los tiempos actuales y futuros, otorga al cooperativismo una relevancia y pertinencia aún mayor para promover la creciente inclusión económica y social y la promoción del desarrollo territorial. Estas nuevas perspectivas, de oportunidades y desafíos al cooperativismo, contemplando la inclusión social y económica, el desarrollo territorial y la sostenibilidad, indican y sugieren prioridades el fortalecimiento del sistema cooperativo, calificación de la gestión, mayores inversiones en la educación de sus miembros, innovación productiva y tecnológica, y la conformación de nuevas y mayores alianzas estratégicas de intercooperación y del sistema cooperativo con la sociedad.

Con las características propias de la región, siendo, por un lado, una región mediterránea, y por el otro, ser de frontera internacional, qué comportamientos podrán surgir a partir de inversiones exógenas y/o cambios en las relaciones políticas, económicas, comerciales y culturales en las relaciones de frontera internacional. El desarrollo futuro de la región será el resultado de la combinación de sus propias competencias estratégicas y tecnológicas involucrando la red institucional presente y las políticas y programas protagonizados por agentes exógenos. La constitución de un sistema de relaciones internas y externas, y de las diversas variables entre sí, debe ser sostenida por inversiones en investigación, ciencia y tecnología, en la formación y capacitación de los agentes y actores locales del desarrollo. Esta dinámica será altamente determinante para constituir un territorio innovador y emprendedor y con una acelerada dinámica de desarrollo, promoviendo el agregado de valor económico, equidad social y sustentabilidad ambiental. 
Pedro Luís Büttenbender, Jorge Oneide Sausen, Bruno Nonnemacher Büttenbender, Ariosto Sparemberger

\section{REFERÊNCIAS}

ALMEIDA, F. Os desafios da sustentabilidade: uma ruptura urgente. Rio de Janeiro: Elsevier, 2007.

BOISIER, S. Em busca do esquivo Desenvolvimento Regional: Entre a Caixa-preta e o projeto político. Revista Planejamento e Políticas Públicas, n 13. Brasília: IPEA, 1996.

BRANDÃO, C. Território e Desenvolvimento: as múltiplas escalas entre o local e o global. São Paulo: Unicamp, 2007.

BENECKE, D.W. Cooperação \& Desenvolvimento. Porto Alegre: Coojornal. 1980.

BRYMAN, A. Quantity and Quality in social research. London, Unwin Hyman, 1988.

BÜTTENBENDER, P.L. VAN DER SAND, A. Plano estratégico de Desenvolvimento da Região Fronteira Noroeste do RS. Corede FN. ljuí/RS: Unijuí, 2017.

BÜTTENBENDER, P.L. Prácticas innovadoras de gestión del desarrollo de región de frontera: el caso de la Región del Noroeste del Estado do Rio Grande do Sul.(tese Doutorado em Administração). FCE/UNaM. 28.08.2014.

. Gestão de Cooperativas. Fundamentos, Estudos e Práticas. Ijuí/RS: Unijuí, 2011.

Arranjos institucionais, Cooperação e Desenvolvimento. Redes econômicas, tecnológicas e sociais, sementes do desenvolvimento e agregação de valor. Ijuí/RS: Unijuí, 2010.

. Estratégias, Inovação e Aprendizagem Organizacional. Cooperação e gestão de competências para o desenvolvimento. Ijuí RS: Unijuí, 2008.

. O cooperativismo e o desenvolvimento regional: estudo sobre as contribuições das cooperativas e das associações no desenvolvimento da região noroeste. Perspectiva Econômica, n. 36. vol. 29, n. 86, São Leopoldo: Unisinos, 1995.

DAGNINO, R. A Relação Universidade-Empresa no Brasil e o "Argumento da Hélice Tripla". Revista Brasileira de Inovação, v.2, n.2, 2003.

DALLABRIDA, V.R. (Org.) Governança territorial e desenvolvimento: descentralização político-administrativa, estruturas subnacionais de gestão do desenvolvimento e capacidades estatais. Rio de Janeiro: Garamond, 2011.

DALLABRIDA, V.R.; ZIMERMANN, V.J.; BÜTTENBENDER, P.L.; BIRKNER, W.M.K.

Gestão Pública Descentralizada e Estruturas Subnacionais de Gestão do 
Desenvolvimento. In: Hermany, R. Gestão local e políticas públicas. Santa Cruz do Sul/RS: Instituto Pe. Reus/UNISC, 2010. p.373-402.

DELGADO, J.M.; GUTIÉRREZ, J. (orgs.). Métodos y técnicas cualitativas de investigación en ciencias sociales. Madri: Síntesis, 1995.

DIAS, R. Gestão Ambiental: responsabilidade social e sustentabilidade. São Paulo: Atlas, 2009.

DRUCKER, Peter. Administrando para o futuro. São Paulo: Thompson, 1998.

EASTERBY-SMITH, M.; THORPE, R; LOWE, A. Management Research: an introduction. London: Sege Publications, 1991.

ELKINGTON, J. Cannibals With Forks: The Triple Bottom Line of 21st Century Business, Stony Creek, CT: New Society Publishers, 1998.

ETZKOWITZ, H. Hélice Tríplice: universidade-indústria-governo: inovação em ação. Porto Alegre: EDIPUCRS, 2009.

FERNÁNDEZ, V.R. Desarrollo regional, espacios nacionales y capacidades estatales: redefiniendo el cenário global-local. Santa Fé (AR): Ediciones UNL, 2003.

FERNÁNDEZ, V.R.; AMIN, A.; VIGIL, J.I. Repensando el desarrollo regional. Contribuciones globales para una estrategia latinoamericana. BAs: Miño y Dávila, 2008.

FIGUEIREDO, P.N. Gestão da Inovação: conceitos, métricas e experiências de empresas no Brasil. Rio de Janeiro: LTC, 2009.

FISCHER, T. Gestão do Desenvolvimento e Poderes Locais: marcos teóricos e avaliação, Salvador: Casa da Qualidade, 2002.

JULIEN, P.A. Empreendedorismo Regional e economia do conhecimento. São Paulo: Saraiva, 2010.

LOPES, R. Competitividade, Inovação e Territórios. Oeiras (PT): Celta, 2001.

MAUERHOFER, V. 3-D Sustainability: an approach for priority setting in situation of conflicting interests towards a sustainable development. Ecological Economics, v. 64, 2008. p. 496-506.

MÉNDEZ, R. Difusión de innovaciones en sistemas productivos locales ydesarrollo territorial. In: III Congreso Internacional de la Red SIAL “Alimentación y Territorios”, Universidad Internacional de Andalucía, España. 2006. 
NICKEL, K.; HASS, F.R.; BÜTTENBENDER, P.L. Estudo sobre a estrutura de governança de uma cooperativa de crédito: O caso da Sicredi União RS. In: Anais CONLAD Congreso Latinoamericano de Administración, 2016, Posadas: UNaM. 2016.

PETERS, G.; PIERRE, J. Governance without Government? Rethinking Public Administration. Journal of Public Administration Research and Theory: J-PART, Vol. 8, No. 2, Apr./1998, pp. 223-243.

POPPER, K.R. Conjecturas e refutações. Tradução de Sérgio Bath. Brasília: UnB, 1982.

ROTTA, E.; BÜTTENBENDER, P.L..; HOFLER, C.E. A presença dos princípios do cooperativismo no Noroeste do Rio Grande do Sul: revisitando a tradição histórica In: Corazza, G.; Radim,J.C. Fronteira Sul: Ensaios socioeconomicos. Porto Alegre : Insular, 2016.

SACHS, I. Desenvolvimento includente, sustentável, sustentado. RJ: Garamond, 2004.

SEN, A. Desenvolvimento como Liberdade. São Paulo: Companhia das Letras, 2000.

SESCOOP/RS. Expressão do Cooperativismo Gaúcho. Porto Alegre/RS. Ed. Sescoop. 2018.

SIEDENBERG, D.R., BÜTTENBENDER, P.L.; ALLEEBRANDT, S.L. Detalhamento de um Modelo de Planejamento Estratégico Terriotiral In: SIEDENBERG, D.R. Fundamentos e Técnicas de Planejamento Estratégico Local/Regional. Santa Cruz do Sul/RS:

Unisc, 2010.

TAYLOR, SJ.; BOGDAN, R. Introducción a los métodos cualitativos de investigación: la búsqueda de significados. Barcelona: Paidós, 1996.

THEIS, I.M. Globalização e planejamento do desenvolvimento regional: o caso do Vale do Itajaí. In: SIEBERT, C. (Org.). Desenvolvimento regional em Santa Catarina: reflexões, tendências e perspectivas. Blumenau: Codesc \& Edifurb, 2001. p. 213-244.

TRIVIÑOS, AN.S. Introdução à Pesquisa em Ciências Sociais. São Paulo, Atlas, 1987.

VAINER, C. As escalas do poder e o poder das escalas: o que pode o poder local? Cadernos do IPPUR, ano XVI, n. 1, Número especial, Planejamento e território: ensaios sobre a desigualdade. 2002.

VEIGA, J.E. Desenvolvimento sustentável. O desafio do Século XXI. SP: Garamond. 2006.

VELTZ, P. Des Territoires pour Apprendre et Innover. Paris: Editions de l’Aube, 1995. 
YIN, R.K. Estudo de caso: planejamento e métodos. Porto Alegre: Bookman, 2005.

Pedro Luís Büttenbender. Doutor em Administração, Mestre em Gestão. Empresarial, Especialista em Cooperativismo em Administração Estratégica. Bacharel em Administração e Tecnólogo. Professor Pesquisador da Unijuí. Rua do Comércio, 3000. .98.700-000. ljuí/RS. pedrolb@unijui.edu.br

Jorge Oneide Sausen. Pós-Doutor em Administração, Doutor em Engenharia da Produção, Bacharel em Administração. Professor pesquisador da Unijuí. josausen@unijui.edu.br

Bruno Nonnemacher Büttenbender. Doutorando em Ambiente e Desenvolvimento no PPGAD/Univates. Bolsista Prosuc/Capes. Mestre em Ambiente e Desenvolvimento no PPGAD/Univates. Graduado em Administração de Empresas pela Unijuí. brunonbuttenbender@gmail.com

Ariosto Sparemberger. Doutor e Mestre em Administração, Bacharel em Administração e Tecnólogo. Professor Pesquisador da Unijuí ariosto@unijui.edu.br

Como citar: BÜTTENBENDER, Pedro Luís et al. Alianzas estratégicas y prácticas cooperativas que promueven la inclusión y el desarrollo territorial: Propuesta de un constructo de gobernanza cooperativa y territorial. Redes (St. Cruz Sul, Online), Santa Cruz do Sul, v. 25, p. 1676-1697, nov. 2020. ISSN 1982-6745. doi:https://doi.org/10.17058/redes.v25i4.14259.

\section{CONTRIBUIÇÃO DE CADA AUTOR}

Pedro Luís Büttenbender:

Contribuições do autor: membro do Grupo de Pesquisa "Gestão Estratégica para o Desenvolvimento" na qualidade de professor pesquisador e membro coordenador do projeto de pesquisa "Estudo sobre as organizações cooperativas do Noroeste Gaúcho, direcionado ao fortalecimento, sustentabilidade e inovação do cooperativismo e suas contribuições para o desenvolvimento regional" que gerou os fundamentos para a elaboração deste artigo. Contribuiu com todas as etapas da pesquisa e da elaboração do Artigo.

Jorge Oneide Sausen:

Contribuições do autor: membro do Grupo de Pesquisa ${ }^{1}$ na qualidade de professor pesquisador, Coordenador do Grupo de Pesquisa e membro do projeto de pesquisa ${ }^{2}$ que gerou os fundamentos para a elaboração deste artigo. Contribuiu com aportes e revisão teórico-conceitual e a problematização e contribuiu com a revisão geral o Artigo. 
Bruno Nonnemacher Büttenbender:

Contribuições do autor: membro do Grupo de Pesquisa ${ }^{1}$ e do Projeto de Pesquisa ${ }^{2}$, na qualidade de Bolsista de Iniciação Científica/Unijuí. Isto quando do período de Coleta e sistematização de dados. Contribuiu com coleta e sistematização de dados, elaboração de figuras ilustrativas deste artigo e de outros resultados do projeto de pesquisa. Atualmente segue como membro voluntário do Projeto de Pesquisa.

Ariosto Sparemberger:

Contribuições do autor: membro do Grupo de Pesquisa ${ }^{1}$, na qualidade de professor pesquisador e membro do projeto de pesquisa² que gerou os fundamentos para a elaboração deste artigo. Contribuiu com aportes e revisão teórico-conceitual, todas as etapas do processo de pesquisa e, em específico com revisão do texto e das referências bibliográficas do Artigo.

Fontes de financiamento: Unijuí; Sescoop/RS. 